ERO code benchmarking of ITER first wall beryllium erosion/re-deposition against LIM predictions

This article has been downloaded from IOPscience. Please scroll down to see the full text article.

2011 Phys. Scr. 2011014008

(http://iopscience.iop.org/1402-4896/2011/T145/014008)

View the table of contents for this issue, or go to the journal homepage for more

Download details:

IP Address: 157.193.59.60

The article was downloaded on 17/02/2012 at 08:55

Please note that terms and conditions apply. 


\title{
ERO code benchmarking of ITER first wall beryllium erosion/re-deposition against LIM predictions
}

\author{
D Borodin ${ }^{1}$, A Kirschner ${ }^{1}$, S Carpentier-Chouchana ${ }^{2}$, R A Pitts ${ }^{2}$, \\ S Lisgo ${ }^{2}$, C Björkas ${ }^{1,3}$, P C Stangeby ${ }^{4}$, J D Elder $^{4}$, A Galonska $^{1}$, \\ D Matveev ${ }^{1,5}$, V Philipps ${ }^{1}$ and U Samm ${ }^{1}$ \\ ${ }^{1}$ Institute of Energy and Climate Research-Plasma Physics, Forschungszentrum Jülich GmbH, \\ Association EURATOM-FZJ, Trilateral Euregio Cluster, Jülich, Germany \\ ${ }^{2}$ ITER Organization, Route de Vinon sur Verdon, 13115 St Paul Lez Durance, France \\ ${ }^{3}$ EURATOM-Tekes, Department of Physics, University of Helsinki, PO Box 43, \\ FI-00014 University of Helsinki, Finland \\ ${ }^{4}$ University of Toronto Institute Aerospace Studies, Toronto, ON, Canada, M3H 5T6 \\ ${ }^{5}$ Department of Applied Physics, Gent University, Rozier 44, B-9000 Gent, Belgium \\ E-mail: d.borodin@fz-juelich.de
}

Received 26 May 2011

Accepted for publication 17 August 2011

Published 16 December 2011

Online at stacks.iop.org/PhysScr/T145/014008

\begin{abstract}
Previous studies (Carpentier et al 2011 J. Nucl. Mater. 415 S165-S169) carried out with the LIM code of the ITER first wall (FW) on beryllium $(\mathrm{Be})$ erosion, re-deposition and tritium retention by co-deposition under steady-state burning plasma conditions have shown that, depending on input plasma parameter assumptions and sputtering yields, the erosion lifetime and fuel retention on some parts of the FW can be a serious concern. The importance of the issue is such that a benchmark of this previous work is sought and has been provided by the ERO code (Pitts et al 2011 J. Nucl. Mater. 415 S957-S964) simulations described in this paper. Provided that inputs to the codes are carefully matched, excellent agreement is found between the erosion/deposition profiles from both codes for a given ITER-shaped FW panel. Issues regarding the difficult problem of the correct treatment of Be sputtering are discussed in relation to the simulations. The possible influence of intrinsic Be impurity is investigated.
\end{abstract}

PACS numbers: 52.40.Hf, 52.65.Pp

(Some figures may appear in colour only in the online journal)

\section{Introduction}

The ITER first wall (FW) comprises 440 blanket modules (BMs), each consisting of a steel-shield block and replaceable FW panels $[1,2]$. Each of these panels is made up of an array of actively cooled fingers, on which Be flat tiles are bonded to provide a low- $Z$ interface to the boundary plasma. The panels are shaped toroidally (and sometimes also poloidally) to protect against panel-to-panel misalignments. An optimized shaping profile is used to minimize the additional heat load penalty under plasma fluxes that such leading edge protection inevitably incurs [3]. The shaping brings another penalty; it creates magnetically shadowed areas in which Be eroded from net erosion areas can be re-deposited. The high-fluence nature of ITER burning plasmas, coupled with the use of a high-erosion-rate material, a non-negligible rate of tritium co-deposition with Be and the FW shaping, means that both erosion lifetime under steady-state plasma fluxes and main chamber fuel retention are potentially serious problems faced by an ITER Be FW. This has been recognized within the ITER Organization, and erosion/re-deposition studies have been conducted for isolated FW panels in regions where plasma-wall interaction is expected to be the most intense. These studies have been conducted with the two-dimensional (2D) LIM guiding centre Monte Carlo (MC) impurity transport code [4]: L(imiter) IM(purity), a limiter version of DIVIMP [5]: DIV(ertor) IMP(urity). The result of this work is a range of erosion lifetimes that vary from acceptable 

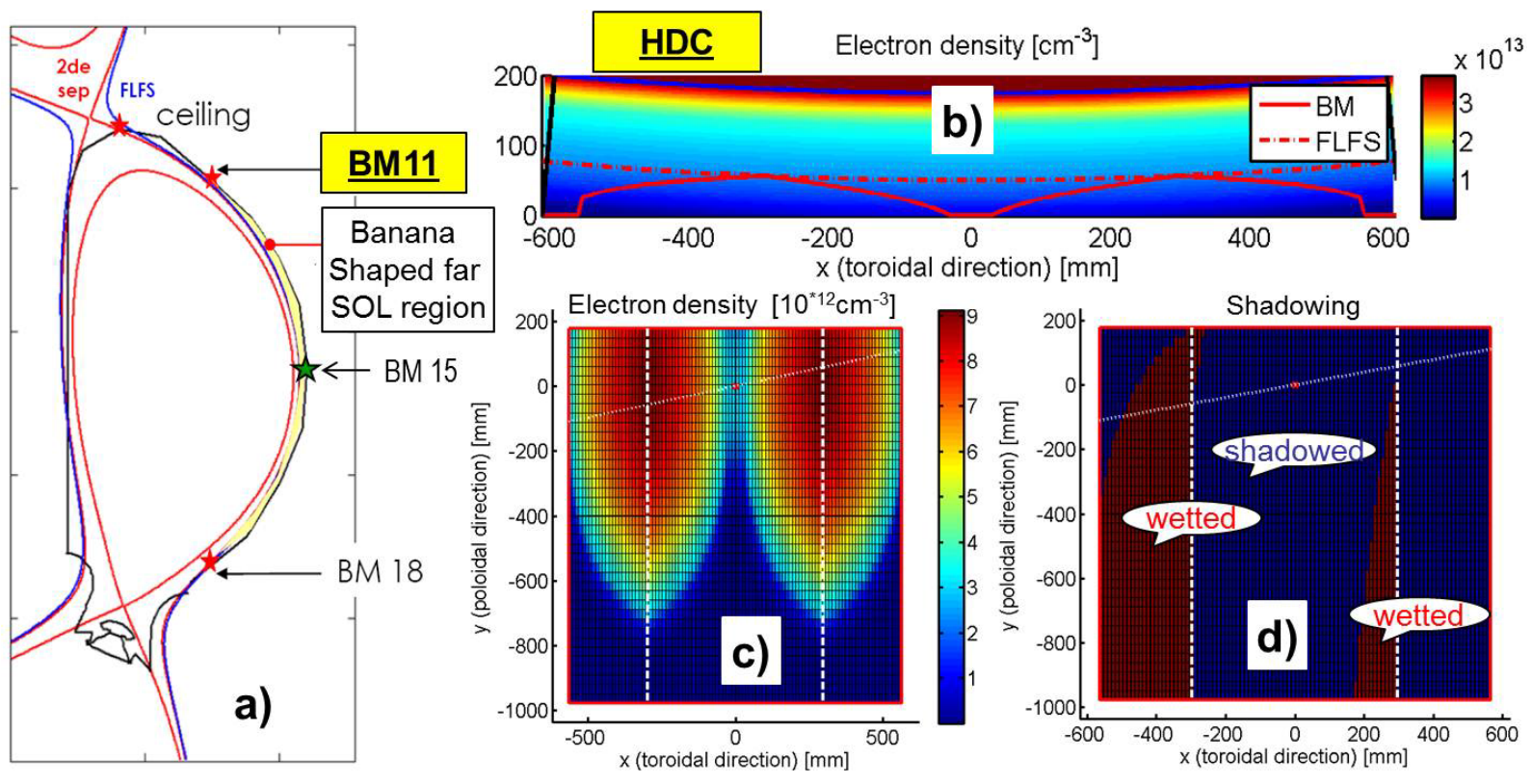

Figure 1. (a) Position of BM 11 on the ITER wall. (b) Electron density (cross-section perpendicular to the BM plane-'side view'). (c) Electron density close to the BM surface ('top view'). (d) Shadowing (as implemented in ERO and LIM) for BM 11 in the HDC case.

throughout the entire planned ITER operational lifetime to posing a rather severe limit $(\sim 1000)$ on the number of burning plasma discharges that could be executed before some FW panels would need to be replaced. Likewise, the associated fuel retention is negligible or will lead to a very rapid accumulation of an FW trapped inventory, comparable in the worst cases to the divertor sink.

While uncertainties in the input plasma parameters and the Be sputtering yields are the main contributing factors to the large range of predicted lifetimes, the potentially important consequences of these findings are such that an independent benchmark of the LIM simulations is required. As reported here, this has now been performed using the ERO [6] impurity transport code, a more recent and more sophisticated simulation tool than the LIM code, which models local erosion and re-deposition in $3 \mathrm{D}$, in comparison with the default 2D mode of LIM and which has previously been applied in the simulation of plasma-surface interaction (PSI) in ITER [7]. The result is an excellent benchmark for equivalent input parameters, providing confidence that the initial LIM simulations are reasonable and allowing some refinements to the modelling with the improved features inherent in ERO.

\section{LIM-ERO benchmark}

ERO is an MC impurity transport and PSI code. It tracks the atoms (and molecules) eroded from a surface and follows their complicated trajectory after ionization (dissociation) determined by friction, anomalous cross-field diffusion and interactions with electromagnetic fields in arbitrary 3D geometry. Sufficient MC test particles are launched in the simulations to guarantee statistically independent results. For example, in the cases reported here, the physical sputtering is represented by 140 test particles starting from each of $20 \times$ 100 cells covering the BM surface. If tracked particles return to the surface, ERO calculates deposition/reflection and possible erosion of additional atoms. If not, the particles are lost from the system. Unlike LIM, which employs the guiding centre approximation, ERO does account for the Larmor gyration of charged particles in magnetic fields (the calculation step is adjusted to guarantee at least seven points per Larmor circle). In the spirit of a code-code benchmark, which this activity represents, wherever possible the input parameters for the ERO runs (plasma parameters, geometry, sputtering yields, etc) have been made identical to those employed in the earlier LIM simulations.

A clear difference between ERO and LIM is in terms of geometry, where the LIM simulations are performed in the 2D toroidal-radial plane in a straight field line approximation such that the toroidal profile of the FW panel is distorted from its real shape. The quasi-3D erosion-re-deposition maps in [4] are thus constructed from multiple toroidal slices assuming no interactions between the individual cuts. By contrast, ERO models the full 3D surface, constructed, in common with LIM, using the analytic shape formulation developed during the BM design [3].

In the simulations, erosion by plasma particles is assumed to occur only on wetted areas of the BMs-however, magnetically shadowed areas can still be accessed by eroded Be which can also induce sputtering there (Be 'self-sputtering'). The erosion by D in the shadowed areas (much smaller than in wetted ones) is neglected because the focus of the paper is the lifetime estimate. ERO generally follows only impurity particles and takes background plasma as the input using the 'test particle approximation'. The shadowed regions are prescribed and computed using an analytic treatment of the combined field line and BM surface geometry, taking into account nearest-neighbour shadowing. The LIM work [4] concentrated on a single BM, BM 11, located near the top low-field side of the poloidal cross-section, where, in the baseline ITER H-mode 15 MA burning plasma scenario, the first limiting flux surface (FLFS)_-just radially outside the second separatrix-intersects the FW (see figure 1(a)). A similar 


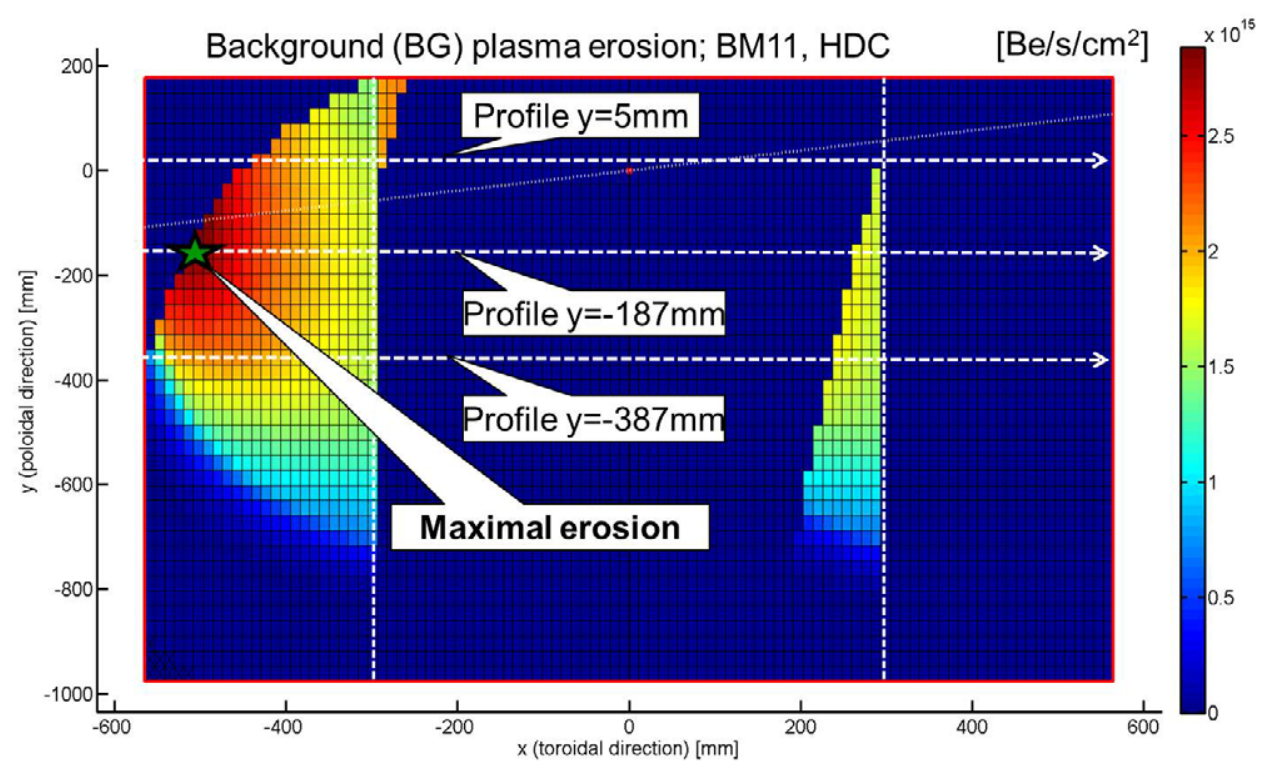

Figure 2. Background plasma erosion of BM 11 in the HDC case and the position of the profiles used for the LIM-ERO benchmark.

intersection point occurs on BM 18 in the lower part of the poloidal cross-section. This limiter-like contact is more appropriate to the situation modelled by LIM (and ERO) of an isolated structure, without the complexity of the secondary $\mathrm{X}$-point region at the top of the main chamber where the most intense 'divertor-like' interactions occur. Since the FLFS is very close to the second separatrix in the ITER baseline equilibrium, the plasma fluxes used as input to the limiter-like modelling are in any case quite close to the (higher) values that would be experienced at the top of the device (e.g. on BM 8 and 9). In terms of the toroidal shaping profile, all panels, regardless of location on the $\mathrm{FW}$, are of similar design, using the same analytic curve to determine the profile shape even though the degree of curvature (edge protection) varies from place to place.

The plasma input fluxes represent, in fact, one of the two main uncertainties in the modelling exercise with LIM or ERO (the other is the Be sputtering yields-see below). For the benchmark, the ERO simulations use the same two sets of flux specifications as for the LIM modelling, namely the 'high' density case (HDC) and the 'low' density case (LDC), which represent the extremes for the far scrape-off layer (SOL) plasma prescribed in the ITER Heat and Nuclear Load Specification document [1], which sets the design heat fluxes for all ITER in-vessel components. Both limits are physics based and describe a low-density SOL (LDC) without significant far SOL convective tails and a high-density variant where turbulent, filamentary transport sets broad-density and flat-temperature profiles extending deeply into the far SOL. The impact on the computed erosion/re-deposition patterns is significant. The influence of transient events (mitigated edge-localized modes (ELM)) is not discussed in this paper as their influence on lifetime was shown by LIM modelling [4] to be negligible in comparison with the steady-state damage.

Regarding sputtering yields, ERO uses angle-averaged sputtering data from [9] assuming a uniform angular distribution and Be ionization data from ADAS '93' exactly as specified in LIM. However, 3D ERO can also make use of more realistic impact angular distribution (see below) and both codes can use more recent ADAS '96' ionization data [8]. Figures 1(b) and (c) illustrate the HDC density contours for both cross-sectional and front surface views on BM 11 together with the prescribed shadowing map on the front surface. At the contact points of the FLFS with the innermost $\mathrm{BM}$ ridges at poloidal location $y=0, n_{\mathrm{e}}=$ $5.7 \times 10^{17} \mathrm{~m}^{-3}, T_{\mathrm{e}}=7 \mathrm{eV}, T_{\mathrm{i}}=18 \mathrm{eV}(\mathrm{LDC})$ and $n_{\mathrm{e}}=1 \times$ $10^{19} \mathrm{~cm}^{-3}, T_{\mathrm{e}}=10 \mathrm{eV}, T_{\mathrm{i}}=20 \mathrm{eV}$ (HDC).

As expected, net erosion occurs in both codes consistent with the input shadow pattern (figure 1(d)). Figure 2 presents the full 2D (toroidal-poloidal) surface erosion map obtained with ERO for the HDC case. Maximum net erosion for HDC occurs at the poloidal location $y=-187 \mathrm{~mm}$ and this is used in figure 3 as the reference location for comparison of ERO and LIM simulations (where, as explained above, the LIM calculations are performed on this single toroidal slice). Evidently, the two codes are in excellent agreement, both for erosion (negative values) and deposition (positive), providing a very satisfactory benchmark. A sticking coefficient of 1 was assumed for deposited Be in LIM, while in ERO, pre-calculated TRIM [9] reflection data are used, although the values are mostly close to unity. The Be self-sputtering in ERO is larger than that in LIM, particularly near the left BM ridge. However, it is only a small fraction $(\sim 20 \%)$ of the total erosion there and elsewhere it is at most only a few per cent, so that the net erosion is still in agreement. The maximum net erosion of $\sim 0.06 \mathrm{~mm} \mathrm{~h}^{-1}$ determines the lifetime of a $1 \mathrm{~cm} \mathrm{Be}$ layer (the approximate thickness of the Be armour foreseen on the ITER FW panels) to be $\sim 167$ plasma hours, which is very well in line with the LIM predictions [4].

For the LDC case (figure 4), the situation is in general similar, although the erosion due to fuel ion impact (background (BG) curves in figure 3) differs more between the two codes than in the HDC runs. A qualitative difference between the results obtained for the two background plasmas (in both ERO and LIM) is a much smaller fraction of eroded Be returning to the surface in the LDC case and thus very low deposition and self-sputtering. At the lower density and temperature of LDC far SOL plasma, most of the eroded 


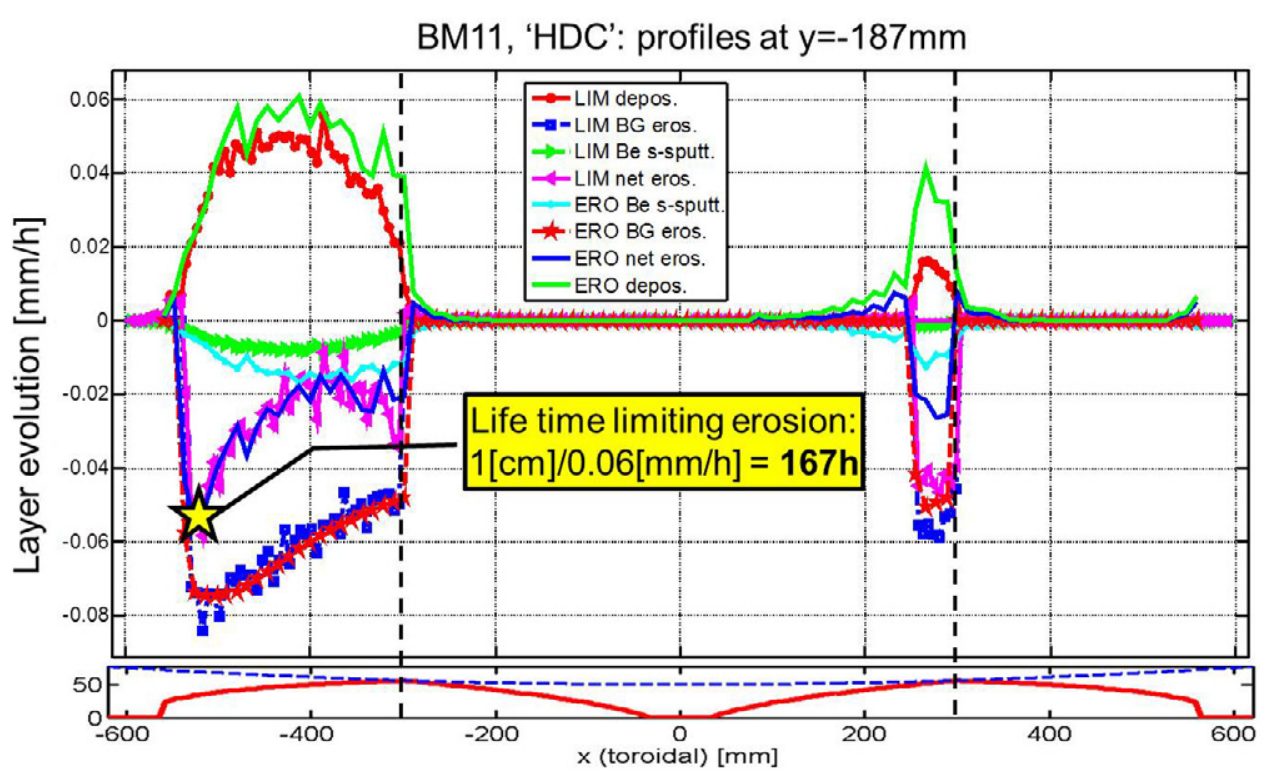

Figure 3. ERO- and LIM-calculated erosion/deposition profiles along BM 11 in the toroidal direction at the position of maximum net erosion ( $y=-187 \mathrm{~cm}$; see figure 2$)$ for the HDC case.

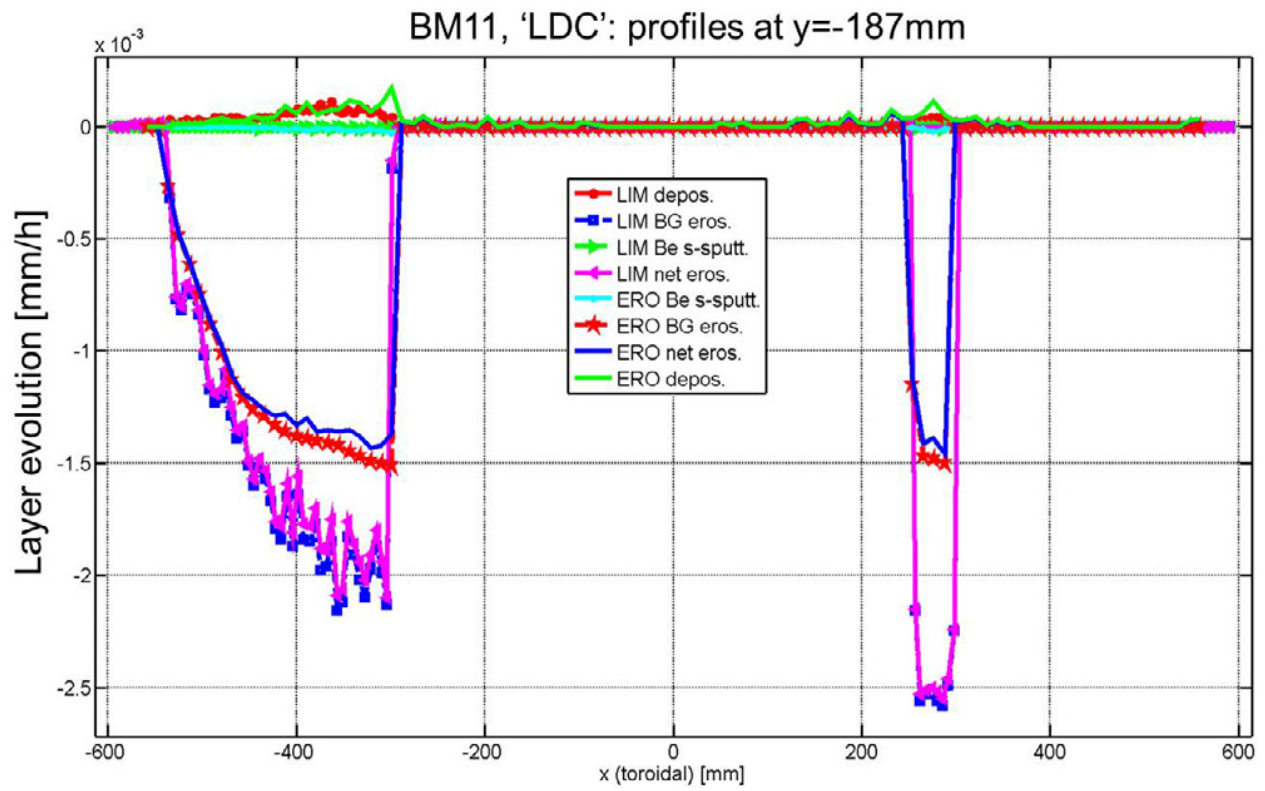

Figure 4. ERO and LIM erosion: re-deposition profiles at $y=-187 \mathrm{~mm}$ for the LDC case.

Be atoms penetrate deeper into the plasma (figure 5) and, finally, leave the ERO simulation volume, mostly in toroidal direction. Similar behaviour is noted in the LIM simulations. Note that the considerably reduced fluxes in the LDC case lead to a greatly extended panel lifetime in comparison with the HDC plasma.

\section{Sputtering yields}

As mentioned above, along with the input plasma parameters, the sputtering yields assumed for both self-sputtering and $\mathrm{D}$ bombardment of Be are a key factor in determining the modelling results. The $\mathrm{D} \rightarrow \mathrm{Be}$ yields have been measured experimentally in several different plasma devices, such as the PISCES-B facility [12], ion beam experiments [14] and JET [15], the only major tokamak to have used Be plasma-facing components. The results, however, vary by order(s) of magnitude. It is also difficult to interpret the particular influence of various parameters-angles of incidence, impurities, surface roughness, etc. Beryllium self-sputtering ( $\mathrm{Be} \rightarrow \mathrm{Be}$ ) has, to our knowledge, not been studied experimentally in the low-energy range of interest in the present simulations. As a consequence, this work uses sputtering yields obtained through simulations, which have the additional advantage of providing angular dependences. Eckstein [10] has provided yields with the help of the SDTrimSP code based on the binary collisional approximation (BCA), and the resulting fit formula in the form of

$$
\boldsymbol{Y}\left(\boldsymbol{E}_{\mathrm{in}}, \boldsymbol{\alpha}\right)=\boldsymbol{Y}\left(\boldsymbol{E}_{\mathrm{in}}, \mathbf{0}^{\mathrm{o}}\right) \times \boldsymbol{A}\left(\boldsymbol{\alpha}, \boldsymbol{E}_{\mathrm{in}}\right),
$$

where $\boldsymbol{E}_{\text {in }}$ and $\boldsymbol{\alpha}$ are, respectively, the energy and angle of incidence. The formula is factorized as a yield for the normal incidence case $\boldsymbol{Y}\left(\boldsymbol{E}_{\mathrm{in}}, \mathbf{0}^{\mathrm{o}}\right)$ and an angular part $\mathbf{A}\left(\boldsymbol{\alpha}, \boldsymbol{E}_{\mathrm{in}}\right)$. 

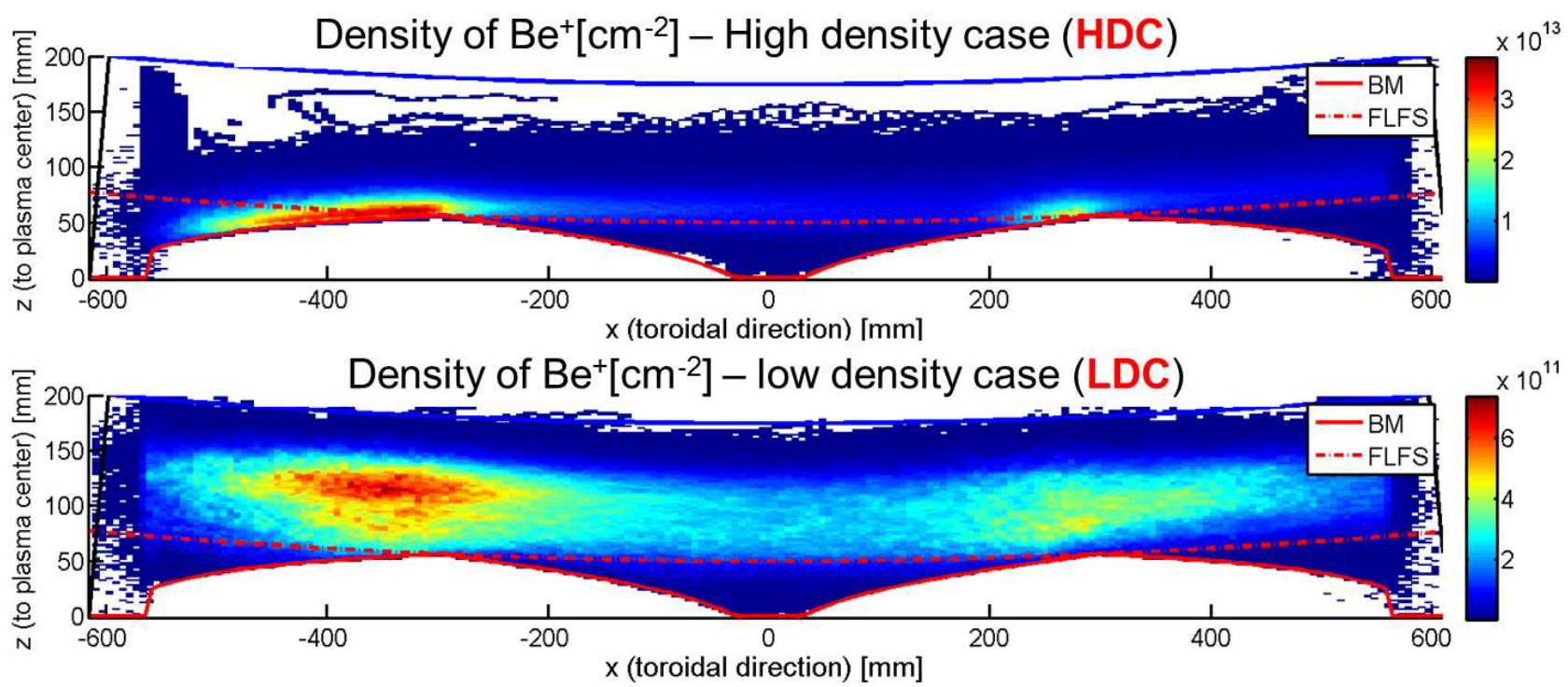

Figure 5. ERO-simulated Be density near BM 11 for the LDC and HDC cases illustrating the variation in penetration depth determined by ionization (affecting re-deposition).

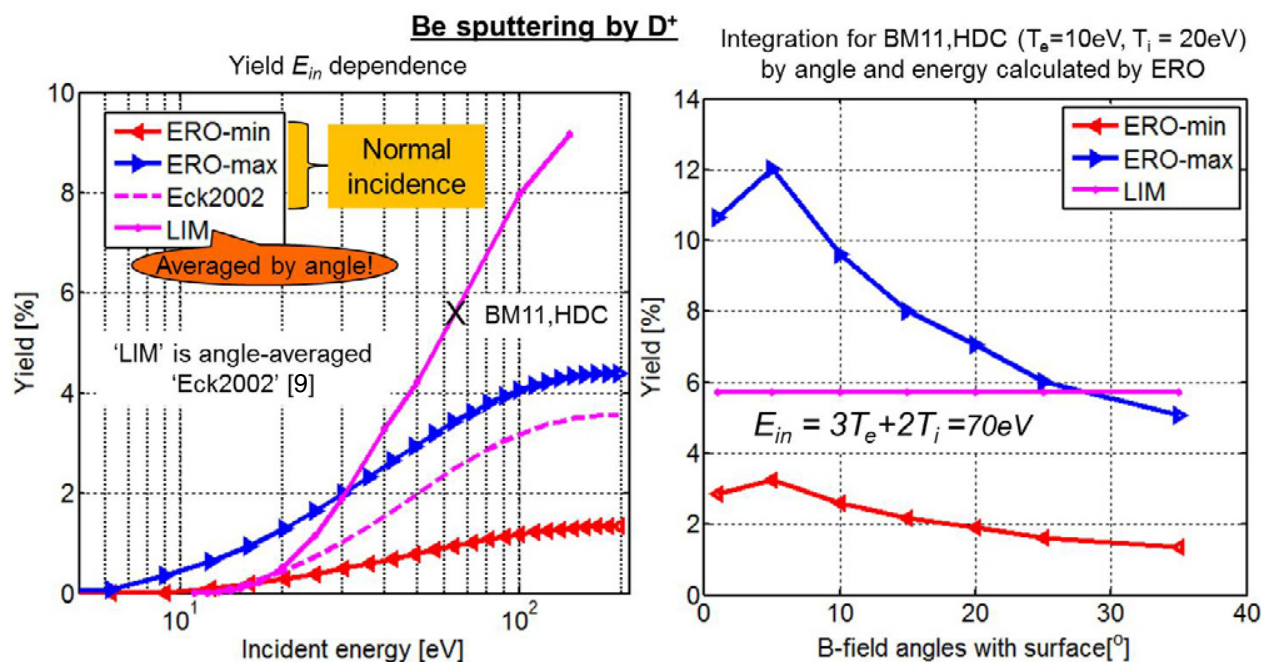

Figure 6. Sputtering yields of Be by $\mathrm{D}^{+}$used in the calculations. 'ERO-min' and 'ERO-max' are the high- and low-limit curves based on a separate simulation study using SDTrimSP. 'LIM' denotes angle-averaged data 'Eck2002' [9] used in the LIM simulations [4] and in ERO for the reference benchmark. ERO uses pre-calculated (in this case for BM 11 and HDC) energy and angle distributions to calculate effective sputtering by background plasma for various angles between the surface and the $B$-field.

One should, however, take into account the fact that the BCA method is not reliable at low energies $(<100 \mathrm{eV})$ and that static SDTrimSP calculations do not account for changes in the hydrogenic concentration in the Be surface (namely D, T in the case of ITER burning plasmas). In an attempt to account for some of these uncertainties, fits have been made to the highest (no deuterium in the surface) and the lowest (50\% deuterium in the surface) calculated yields for normal incidence using SDTrimSP. In the case of D bombardment, for example, the maximum allowed D content (varied up to 50\%) in the sample and the respective yields from the SDTrimSP simulations are then compared with the data derived from molecular dynamics simulations [11], where the hydrogenic content is taken into account by performing cumulative simulations. (The details of these calculations will be reported elsewhere.) The minimum and maximum yields for $\mathrm{D}^{+}$and $\mathrm{Be}^{\mathrm{n}+}$ on $\mathrm{Be}$ at normal incidence
$\mathbf{Y}\left(\boldsymbol{E}_{\text {in }}, \boldsymbol{0}^{\circ}\right)$ obtained this way are denoted by 'ERO-min' and 'ERO-max', respectively (see figure 6, left). The formula and parameters of Eckstein [10] have been used to provide the angular-dependent sputtering yields as illustrated in figure 6 (right).

These ERO 'pre-calculated' $\boldsymbol{E}_{\text {in }}$ and $\boldsymbol{\alpha}$ distributions are used for both $\mathrm{D}^{+}$and intrinsic $\mathrm{Be}^{\mathrm{n}+}$ plasma impurities to calculate the effective sputtering yield by the background plasma as a function of $B$-field angle to the surface (figure 6 , right).

For eroded Be particles, which the MC code follows from birth, $\boldsymbol{E}_{\text {in }}$ and $\boldsymbol{\alpha}$ are known, so the fit is used directly. In both cases, the angular part $\mathbf{A}\left(\boldsymbol{\alpha}, \boldsymbol{E}_{\text {in }}\right)$ is obtained using the calculated fitting parameters (four values for each $\boldsymbol{E}_{\text {in }}$ ) from [10]. In the LIM simulations, the older SDTrimSP data [9] are used, angle averaged assuming a uniform angle of incidence distribution. Figure 6 (left) shows that the minimum 


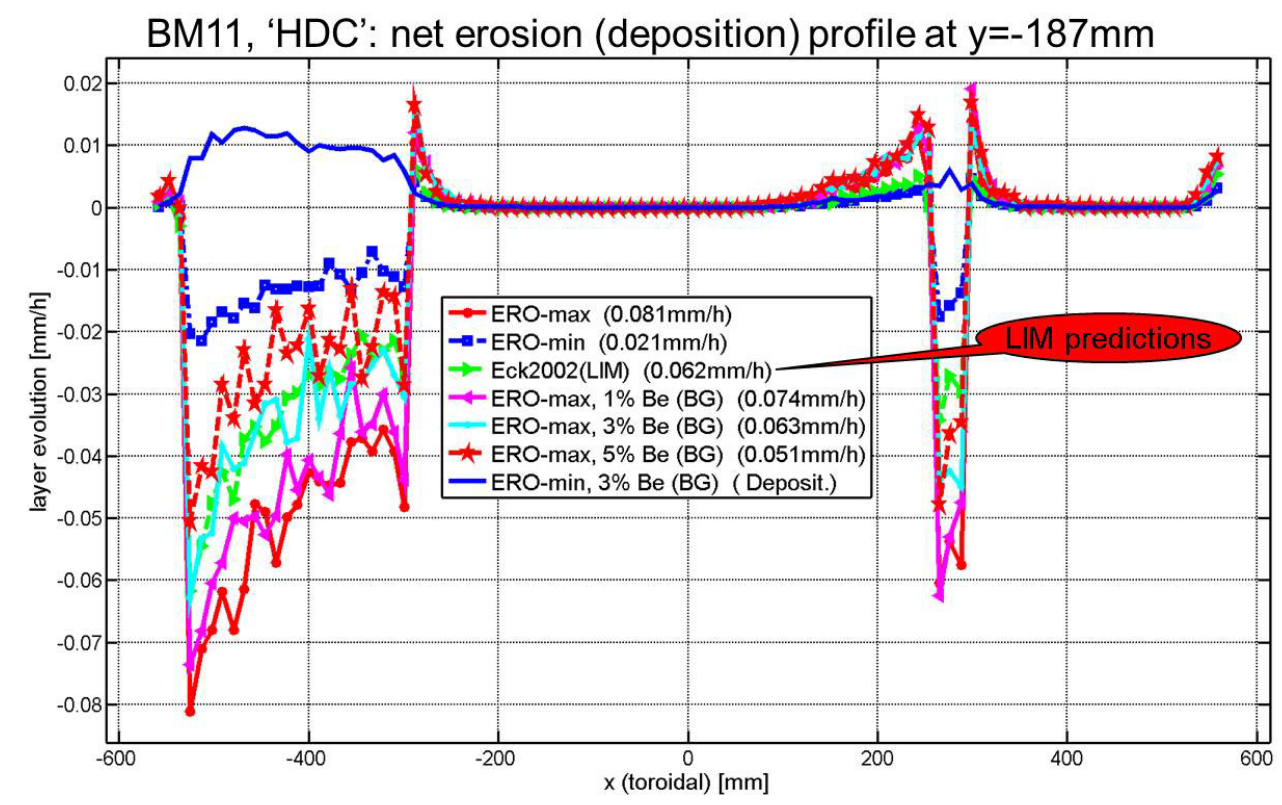

Figure 7. ERO-simulated net erosion (as in figure 2) for a variety of assumptions on sputtering yield and intrinsic Be impurity concentration.

and maximum yields differ by a factor of 3-5 in the relevant energy range.

\section{Parameter study and discussion}

Figure 7 compiles the net erosion/deposition profiles for the HDC case at the reference poloidal location of maximum erosion $(y=-187 \mathrm{~mm})$ for a variety of assumptions regarding the sputtering yields and an additional element compared with the LIM simulations, the influence of intrinsic $\mathrm{Be}$ in the incoming plasma fluxes. For each assumption, the peak erosion rate (which determines the lifetime) on the profile is given in the legend. The 'Eck2002(LIM)' curve is essentially the same as 'ERO net eros.' profile in figure 3, with a minor difference coming from the use of more recent ADAS '96' data for Be ionization. The 'ERO-max' assumption produces an increase of the reference erosion maximum by $\sim 30 \%\left(0.08 \mathrm{~mm} \mathrm{~h}^{-1}\right.$ versus $\left.0.06 \mathrm{~mm} \mathrm{~h}^{-1}\right)$, thus providing the most 'pessimistic' lifetime in the framework of these isolated BM simulations. Assuming the 'ERO-min' yields, the erosion $\left(0.02 \mathrm{~mm} \mathrm{~h}^{-1}\right)$ is reduced by a factor of $\sim 3$ compared with the reference case. For the baseline ITER H-mode discharge, with a burning plasma flat top of $400 \mathrm{~s}$, the end of life for a $10 \mathrm{~mm}$ Be thickness would thus be reached somewhere in the range of 1100-4200 discharges if the far SOL plasma is as specified in the ITER thermal load prescriptions.

The presence of Be impurity in the background plasma produces two opposing effects: (a) deposition of additional Be and (b) increased Be erosion due to self-sputtering. To provide a first crude estimate of the effect, a fixed concentration of $\mathrm{Be}^{+}$in the incident $\mathrm{D}^{+}$flux to the surface is assumed. The ERO modelling shows (figure 7) that at least for the case at hand, effect (a) dominates. Intrinsic Be impurity decreases the 'ERO-max'-based net erosion by up to $40 \%$ if $5 \%$ Be impurity is assumed. Assumptions of 3 and $1 \%$ Be lead logically to an intermediate effect. A more realistic treatment would assume a decrease in the concentration gradient in moving away from the BM surface, using, for example, a specified e-folding distance. Similar calculations have already been performed with ERO for background carbon impurity in TEXTOR [16] to match experimental data. Carbon concentrations of a few per cent were required to obtain agreement.

It is interesting to note from figure 7 that a combination of low erosion ('ERO-min') and 3\% intrinsic Be impurity leads to pure deposition. This is of course unrealistic, since it contradicts the overall surface-plasma particle balance. However, it does demonstrate that parameter scans can identify reasonable limits for the input assumptions (at least within this simple situation of an isolated BM).

It should also be clear that there are many additional factors in the physics model which could influence the erosion-deposition maps obtained here (e.g. the local flow velocity, sheath potential, surface temperature, etc). Additional surface effects, already introduced in previous ERO studies, such as enhanced re-erosion [17] and Be carbide formation [18], or new processes yet to be studied (e.g. the formation of Be-D molecules [19]) may also play a role. Faced, however, with the very large current uncertainty in the expected plasma background and the spread of possible sputtering yields, it is likely that most of these effects will provide only second order corrections.

The code-code comparisons shown in the present work now need to be reinforced by a true experimental benchmark, under relevant and known SOL plasma conditions and in a majority Be environment. The rather pessimistic lower limits for the erosion lifetime under ITER conditions found with these simulations increases the importance of such a benchmark. Future experiments on the JET ITER-like wall [20] will hopefully provide useful input. It is also worth mentioning that the present work is in many aspects similar to the modelling, code-code bechmarking (ERO-EDDY) and parameter studies using ERO, which have been performed for erosion and re-deposition at the ITER divertor [21]. 


\section{Summary}

The use of low-Z, high-sputtering-yield Be on the ITER FW, coupled with a shaping design that avoids plasma-exposed edges on FW panels, is a concern for erosion lifetime under the high-fluence conditions that parts of the main chamber will experience during long-pulse, burning plasma operation. Predictive modelling of this erosion (and associated re-deposition) for an isolated panel on the $\mathrm{FW}$ near the region of most intense plasma interactions was first performed in 2D using the LIM MC impurity transport code. This has now been carefully benchmarked, as described in this paper, against the ERO code, a more recent simulation tool based on the same principles as LIM, but incorporating a full gyro-orbit description and with default 3D geometry. The result of the benchmark, when all possible input parameters (plasma fluxes, panel geometry, sputtering yields and magnetic shadowing) are made equivalent in the two codes, is excellent agreement for the surface Be erosion-re-deposition pattern and the absolute erosion lifetimes. As in the LIM simulations, ERO therefore predicts that for the highest density plasma case, the peak erosion rates on the simulated FW panel are such that end-of-life for a Be armour thickness of $1 \mathrm{~cm}$ would be reached in $\sim 166$ plasma exposure hours, or roughly 1500 reference $\mathrm{H}$-mode discharges at $Q=10$ and $\sim 400$ s duration. The influence of transient events previously estimated by LIM [1] would be taken into account by ERO in a similar way, so they are meaningless for the benchmark.

The ERO study has also taken into account more refined assumptions regarding the sputter yield dependence (angle and energy) and introduced various concentrations of intrinsic Be into the incoming plasma flux. The result of variations in the sputter yields is a further uncertainty in the erosion rate, with the lower limits for the highest-density plasma case falling in the range 1100-4200. The inclusion of intrinsic Be in the background plasma can provide a significant correction to these values, decreasing the net erosion by a factor of up to $40 \%$ depending on the Be concentration.

A number of other physics and surface effects can be included in further simulations, but it is likely that these will be of second order in comparison with the uncertainties introduced by variations in the sputter yields and input plasma parameters. Given the potentially important consequences for ITER operation of the LIM-ERO-predicted lower lifetime limits in some areas of the main chamber wall, it would seem prudent to proceed with an experimental benchmark of these simulations. The JET ITER-like wall [20] should provide an excellent opportunity.

\section{Acknowledgments}

The work was supported by the EU Task Force on Plasma-Wall Interaction in the framework of the ITPA collaboration. Computer time for computations was provided by the Jülich Supercomputer Centre. The authors are grateful to R Doerner, S Brezinsek, B Unterberg and M Tokar for fruitful discussions and to W Eckstein and A Mutzke for assistance with SDTrimSP calculations.

Published under license from EURATOM.

\section{References}

[1] Pitts R A et al 2011 J. Nucl. Mater. 415 S957-S964

[2] Mitteau R et al 2011 J. Nucl. Mater. 415 S969-S972

[3] Stangeby P C 2009 J. Nucl. Mater. 390/391 963-6

[4] Carpentier S et al 2011 J. Nucl. Mater. 415 S165-S169

[5] Stangeby P C and Elder J D 1992 J. Nucl. Mater. 196-198 258

[6] Kirschner A et al 2000 Nucl. Fusion 40989

[7] Kirschner A et al 2007 J. Nucl. Mater. 363-365 91

[8] Summers H P 2004 The ADAS User Manual version 2.6, http://adas.phys.strath.ac.uk

[9] Eckstein W et al 2002 IPP Report IPP 9/132

[10] Eckstein W 2007 Topics Appl. Phys. 110 33-187

[11] Björkas C et al 2009 New J. Phys. 11123017

[12] Nishijima D et al 2009 J. Nucl. Mater. 390/391 132-5

[13] Schwarz-Selinger T et al 2011 private communication

[14] Roth J et al 1997 Fusion Eng. Des. 37 465-80

[15] Stamp M F et al 2011 J. Nucl. Mater. 415 S170-S173

[16] Droste S et al 2008 Plasma Phys. Control. Fusion 50015006

[17] Kirschner A et al 2004 J. Nucl. Mater. 328 62-6

[18] Borodin D et al 2011 J. Nucl. Mater. 415 S219-S222

[19] Nishijima D et al 2008 Plasma Phys. Control. Fusion 50125007

[20] Brezinsek S et al 2011 J. Nucl. Mater. 415 S936-S942

[21] Kirschner A et al 2009 Phys. Scr. T128 014011 